\title{
The two-dimensional, finite, gas lubricated, slider bearing in slip flow
}

\author{
E. Aliu* J. J. Shepherd ${ }^{\dagger}$
}

(Received 18 December 2005; revised 14 August 2006)

\begin{abstract}
Consider the pressure field in a two dimensional, finite, gas lubricated, slider bearing under so-called 'slip flow' conditions; that is, the bearing gap clearance is comparable to the mean free path distance of the lubricant gas in the bearing. In such cases, a modified Reynolds equation determines the pressure. For high speed flow and a large breadth to width ratio, the resulting boundary value problem for the pressure involves two large parameters and is a singular perturbation problem in the infinite limit of these quantities. Analysis by perturbation techniques yields an approximate expression for the pressure throughout the bearing, displaying side and trailing edge boundary layers. The results of using this expression are compared with those
\end{abstract}

${ }^{*}$ School of Mathematical \& Geospatial Sciences, RMIT University, Melbourne, Australia. mailto: elmas.aliu@rmit.edu.au

${ }^{\dagger}$ School of Mathematical \& Geospatial Sciences, RMIT University, Melbourne, Australia. mailto: jshep@rmit.edu.au

See http://anziamj.austms.org.au/V47EMAC2005/Aliu for this article, (C) Austral. Mathematical Soc. 2006. Published December 15, 2006. ISSN 1446-8735 
obtained from application of a commercial finite element computer package.

\section{Contents}

1 Introduction

C476

2 Perturbation analysis

C478

3 Trailing edge layer: analysis in Region 1

C480

4 Side layers: analysis in Regions 2 and 3

C484

5 Overall representation for the pressure

C486

6 Discussion

C487

References

C491

\section{Introduction}

The bearing geometry is shown in Figure 1 , with the $X$ - and $Z$-axes in the plane of the moving surface, the $X$-axis in the direction of relative motion, and the $Y$-axis perpendicular to the base plane.

The upper surface, $Y=H(X, Z)$, for a given positive function $H$, is stationary, while the lower surface, (the $X Z$-plane), has constant velocity $U$ in the positive $X$ direction.

Under the assumptions of isothermal slip flow (that is, when the bearing gap is comparable in dimension to the mean free path distance of the gas lubricant), and with dimensionless variables $p, h, x$ and $z$ defined by $P=P_{a} p$, 


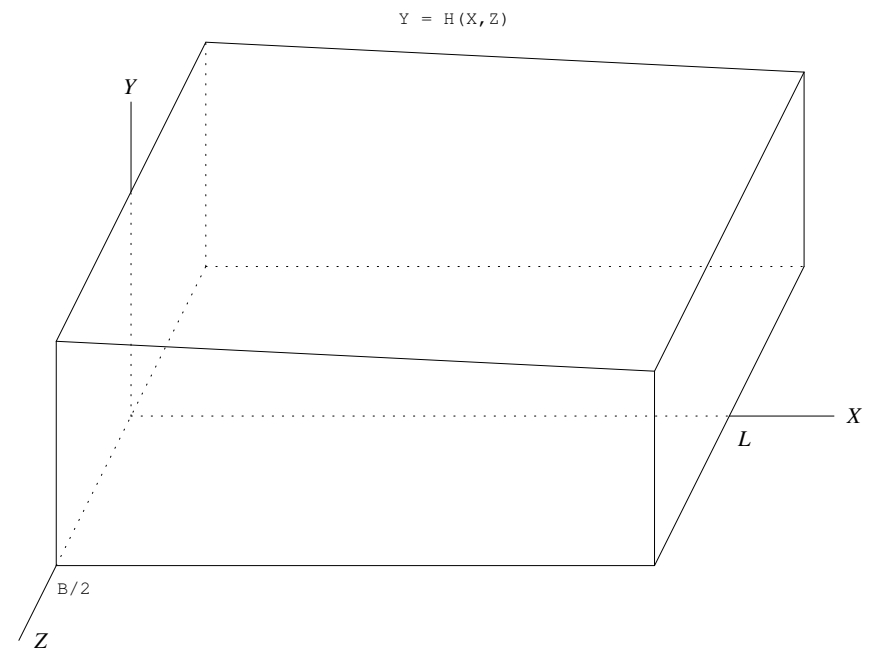

Figure 1: Finite bearing geometry.

$H=H_{0} h, X=L x, Z=B L z$, the task of determining the dimensionless pressure $p$ in the bearing reduces to solving the nonlinear equation

$$
\Lambda^{-1} \frac{\partial}{\partial x}\left[h^{3} p\left(1+\frac{6 k}{h p}\right) \frac{\partial p}{\partial x}\right]+B^{-2} \Lambda^{-1} \frac{\partial}{\partial z}\left[h^{3} p\left(1+\frac{6 k}{h p}\right) \frac{\partial p}{\partial z}\right]=\frac{\partial}{\partial x}(h p),
$$

on $0<x<1,-1 / 2<z<1 / 2$, subject to the boundary conditions

$$
\begin{aligned}
p(x, \pm 1 / 2, \Lambda, B, k) & =1, & & 0 \leq x \leq 1, \\
p(0, z, \Lambda, B, k)=p(1, z, \Lambda, B, k) & =1, & & -1 / 2 \leq z \leq 1 / 2 .
\end{aligned}
$$

Equation (1) is a modification of the Reynolds equation [4], derived by Burgdorfer [3] to deal with the slip flow occurring in the bearing gap under rarefied gas conditions. The dimensionless parameters are the bearing number $\Lambda$ (associated with the flow speed, characterized by $U$ ); the Knudsen number $k$ (associated with the degree of slip in the flow-thus $k=0$ 
signifies zero slip); and the breadth ratio $B$ (giving the ratio of bearing width to depth). $P_{a}$ is the pressure external to the bearing, assumed constant. Thus the boundary conditions (2) and (3) signify the pressure returning to ambient at the boundaries. $H_{0}$ is a characteristic (constant) value of the function $H(X, Z)$.

\section{Perturbation analysis}

The finite but wide high speed isothermal gas slider bearing in slip flow is typified by the conditions

$$
B \gg 1, \quad \Lambda \gg 1, \quad k \approx \mathcal{O}(1) .
$$

Thus a perturbation analysis of the problem (1), (2) and (3) based on the limits $B \rightarrow \infty, \Lambda \rightarrow \infty$ for given $\mathcal{O}(1)$ values of $k$ is indicated. Since two large parameters are involved, $B$ and $\Lambda$, and since, in the most general situation, they may become infinitely large independently, we place a restriction on the limiting process. Thus, we assume that, as $B, \Lambda \rightarrow \infty$,

$$
B^{2} \Lambda^{-1} \rightarrow 0 ;
$$

that is, $B^{-2} \rightarrow 0$ more slowly than $\Lambda^{-1}$. This fits in well with realistic values for practical applications; for example, $B \approx 10, \Lambda \approx 100-2000$, and allows construction of a useful approximate expression for the pressure field.

The reduced equation, obtained by setting $B=\Lambda=\infty$ in (1), is first order in $x$; and thus can meet only one of the boundary conditions (3) (and neither of (2)). Thus, the problem (1), (2) and (3) is a singular perturbation problem as $\Lambda, B \rightarrow \infty$; and appropriate methods of analysis are needed.

Early calculations [2] indicate that the solution of the reduced equation satisfies the boundary condition at $x=0$; but not the boundary conditions at the trailing edge $x=1$ and the side edges $z= \pm \frac{1}{2}$. At these edges, local 


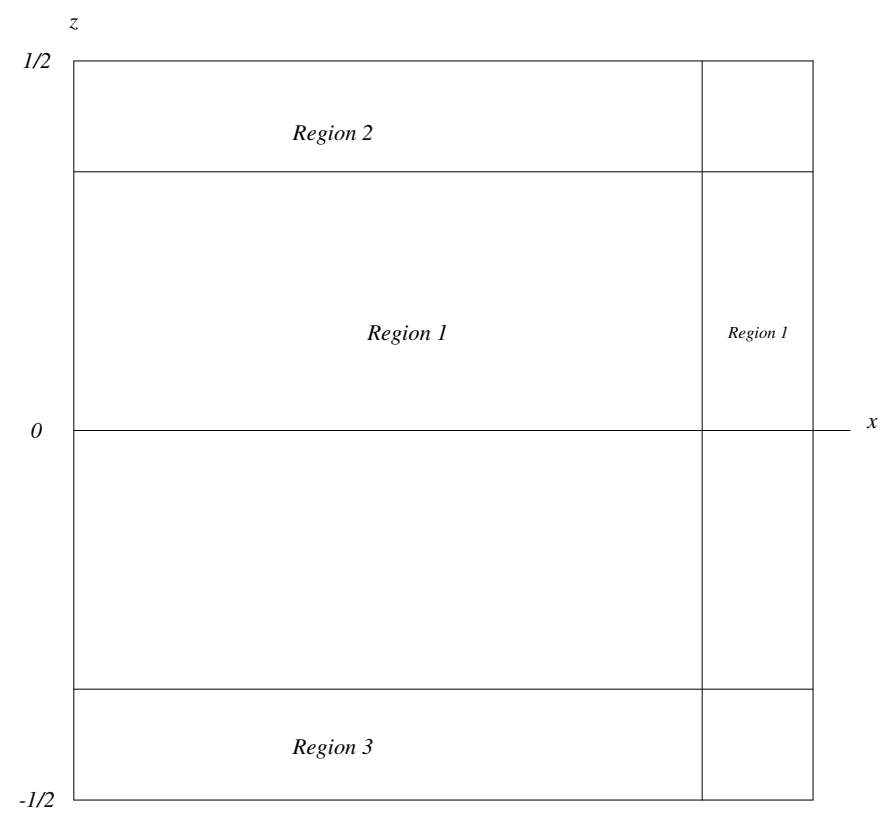

Figure 2: Asymptotic regions for the finite bearing .

boundary layers occur, where the pressure corrects rapidly from the solution of the reduced equation to the prescribed boundary conditions. To analyze these layer solutions, local variables are introduced, and the limit $\Lambda, B \rightarrow \infty$ considered in terms of these variables. Standard arguments $[5,6]$ give the boundary layer thickness at the trailing edge $x=1$ to be $\mathcal{O}\left(\Lambda^{-1}\right)$; whereas the side boundary layers at the edges $z=-\frac{1}{2}$ and $z=\frac{1}{2}$ are of thickness $\mathcal{O}\left(B^{-1} \Lambda^{-1 / 2}\right)$ as $\Lambda, B \rightarrow \infty$.

Perturbation techniques are applied separately in the different sub-regions of the bearing, as shown in Figure 2. The pressure field in Region 1 will be governed by an expansion away from the side boundary layers that incorporate the trailing edge boundary layer adjacent to $x=1$; whereas in Regions 2 and 3 , the analysis includes the side boundary layers adjacent to $z=-\frac{1}{2}$ 
and $z=\frac{1}{2}$ respectively. The findings of Aliu [1] for the one-dimensional case, where the Method of Multiple Scales provided a more accurate approximation than that generated by Matched Expansions, supports an analysis paralleling that of Aliu [1] away from side layers, so that, in Region 1, the problem is analyzed by the Method of Multiple Scales. However, side layer solutions, in Regions 2 and 3, are constructed using the Method of Matched Expansions and linked to the Multiple Scales solution in Region 1 by the standard matching process.

\section{Trailing edge layer: analysis in Region 1}

By analogy with [1], the pressure field in this region, namely $p(x, z, \Lambda, B)=$ $p_{1}(x, z, \Lambda, B)$ is viewed as depending on the "slow" variables $x$ and $z$, as well as the "fast" variable $\xi$, where

$$
\xi=(1-g(x, z)) \Lambda
$$

The function $g$ is to be positive, with other properties as required for the subsequent analysis.

A multiscaling approach argues that the solution of (1) in Region 1 may be viewed as a function $P_{1}(x, z, \xi, \Lambda, B)$ of the three variables $x, z$ and $\xi$, with $\Lambda$ and $B$ as parameters.

Equation (1) transforms to the partial differential equation for $P_{1}$,

$$
\begin{aligned}
& \Lambda^{-2} \frac{\partial}{\partial x}\left[h^{2}\left(h P_{1}+6 k\right) \frac{\partial P_{1}}{\partial x}\right]-\Lambda^{-1} \frac{\partial}{\partial x}\left[h^{2} g_{x}\left(h P_{1}+6 k\right) \frac{\partial P_{1}}{\partial \xi}\right] \\
& -\Lambda^{-1} \frac{\partial}{\partial \xi}\left[h^{2} g_{x}\left(h P_{1}+6 k\right) \frac{\partial P_{1}}{\partial x}\right]+h^{2} g_{x}^{2} \frac{\partial}{\partial \xi}\left[\left(h P_{1}+6 k\right) \frac{\partial P_{1}}{\partial \xi}\right] \\
& +\Lambda^{-2} B^{-2} \frac{\partial}{\partial z}\left[h^{2}\left(h P_{1}+6 k\right) \frac{\partial P_{1}}{\partial z}\right]-\Lambda^{-1} B^{-2} \frac{\partial}{\partial z}\left[h^{2} g_{z}\left(h P_{1}+6 k\right) \frac{\partial P_{1}}{\partial \xi}\right]
\end{aligned}
$$




$$
\begin{aligned}
& -\Lambda^{-1} B^{-2} \frac{\partial}{\partial \xi}\left[h^{2} g_{z}\left(h P_{1}+6 k\right) \frac{\partial P_{1}}{\partial z}\right]+B^{-2} g_{z}^{2} h^{2} \frac{\partial}{\partial \xi}\left[\left(h P_{1}+6 k\right) \frac{\partial P_{1}}{\partial \xi}\right] \\
& =\Lambda^{-1} \frac{\partial}{\partial x}\left(h P_{1}\right)-h g_{x} \frac{\partial P_{1}}{\partial \xi}
\end{aligned}
$$

where subscripts denote partial derivatives. Equation (6) is the multiscaled form of Equation (1). In (6), the function $h$ is to be regarded as depending on the "slow" variables $x$ and $z$ only; and (6) is assumed valid, not only on $0 \leq x \leq 1,-1 / 2<z<1 / 2$, but also on $0 \leq \xi<\infty$. In view of (4), it is assumed that $P_{1}$ has an expansion of the form

$$
P_{1}(x, z, \xi, \Lambda, B)=P_{10}(x, z, \xi)+B^{-2} P_{11}(x, z, \xi, B)+\Lambda^{-1} P_{12}(x, z, \xi)+\cdots .
$$

The form (7) is dictated by the gauge functions displayed in (6) (powers and products of $\Lambda^{-1}$ and $B^{-2}$ ). Note that (7) is not of Poincaré form since the second term (in which $P_{11}$ depends on $B$ ) might be expected to incorporate all terms involving powers of $B^{-2}$ that are lower order than $\mathcal{O}\left(\Lambda^{-1}\right)$.

For the expansion (7), the usual multiple scaling boundedness criterion is to be interpreted as the requirement that the ratios $P_{11} / P_{10}, P_{12} / P_{10}, P_{12} / P_{11}$ all be bounded for small $B^{-2}$ and $\Lambda^{-1}$ uniformly with respect to $0 \leq x \leq 1$, $-1 / 2 \leq z \leq 1 / 2$, and $0 \leq \xi<\infty$.

Substitution of (7) into (6) and equating like orders to zero yields a sequence of partial differential equations for $P_{10}, P_{11}, P_{12}, \ldots$.

The $\mathcal{O}(1)$ terms give the nonlinear equation

$$
h^{2} g_{x}^{2} \frac{\partial}{\partial \xi}\left[\left(h P_{10}+6 k\right) \frac{\partial P_{10}}{\partial \xi}\right]+h g_{x} \frac{\partial P_{10}}{\partial \xi}=0 ;
$$

the $\mathcal{O}\left(B^{-2}\right)$ term gives the linear equation

$$
L\left[P_{11}\right] \equiv h^{3} g_{x}^{2} \frac{\partial^{2}}{\partial \xi^{2}}\left[\left(P_{10}+\frac{6 k}{h}\right) P_{11}\right]+h g_{x} \frac{\partial P_{11}}{\partial \xi}
$$




$$
=-h^{3} g_{z}^{2} \frac{\partial}{\partial \xi}\left[\left(P_{10}+\frac{6 k}{h}\right) \frac{\partial P_{10}}{\partial \xi}\right]
$$

whereas the $\mathcal{O}\left(\Lambda^{-1}\right)$ terms give

$$
\begin{aligned}
L\left[P_{12}\right]= & \frac{\partial}{\partial x}\left(h P_{10}\right)+\frac{\partial}{\partial x}\left[h^{3} g_{x}\left(P_{10}+\frac{6 k}{h}\right) \frac{\partial P_{10}}{\partial \xi}\right] \\
& +\frac{\partial}{\partial \xi}\left[h^{3} g_{x}\left(P_{10}+\frac{6 k}{h}\right) \frac{\partial P_{10}}{\partial x}\right] .
\end{aligned}
$$

Integration of (8) gives the general solution $P_{10}(x, z, \xi)$ implicitly in the form

$$
\begin{aligned}
& P_{10}(x, z, \xi)+\left[F(x, z)+\frac{6 k}{h(x, z)}\right] \ln \left[F(x, z)-P_{10}(x, z, \xi)\right] \\
& =-\frac{\xi}{h^{2} g_{x}}+G(x, z)
\end{aligned}
$$

with $F$ and $G$ functions of $x$ and $z$ alone. We assume that $F(x, z)$ and $G(x, z)$ are continuously differentiable with respect to their arguments, and that $F(x, z)$ and $g_{x}(x, z)$ may be chosen to be positive functions on $0 \leq x \leq 1$, $-1 / 2 \leq z \leq 1 / 2$, whereas $F(x, z)-P_{10}(x, z, \xi) \neq 0$ for all $\xi$ on $0 \leq \xi \leq \infty$. Thus, all quantities in (11) are defined.

Now, (11) may be rearranged as

$$
F(x, z)-P_{10}(x, z, \xi)=\exp \theta,
$$

where

$$
\theta(x, z, \xi)=\frac{h\left(G-P_{10}-g_{x} \xi\right)}{h F+6 k} .
$$

Equations (12) and (13) show that, under the assumptions about $g$ and $F$, $P_{10}$ as defined by (11) has the property that $P_{10}(x, z, \xi) \rightarrow F(x, z)$ monotonically as $\xi \rightarrow \infty$, for all $0 \leq x \leq 1,-1 / 2 \leq z \leq 1 / 2$ and $k \geq 0$. 
Applying the representation (12) for $P_{10}$ gives Equation (10) for $P_{12}$ in the form

$$
\begin{aligned}
& L\left[P_{12}\right]=\frac{\partial}{\partial x}(F h)+e^{\theta}\left(1-\frac{h}{F h+6 k} e^{\theta}\right)^{-1} \\
& \left\{h \frac{\partial}{\partial x}\left[\frac{h(G-F)}{F h+6 k}\right]-h \frac{\partial}{\partial x}\left[\frac{1}{h g_{x}(F h+6 k)}\right] \xi+h \frac{\partial}{\partial x}\left[\frac{1}{F h+6 k}\right]\right. \\
& \left.\quad-\frac{6 k h}{F h+6 k} h_{x}+h\left[\frac{h_{x}}{F h+6 k}+\frac{\partial}{\partial x}\left(\frac{h}{F h+6 k}\right)\right] e^{\theta}\right\}
\end{aligned}
$$

For large $\xi, P_{10} \approx F(x, z)$, and the linear differential operator $L$, Equation (14) is transformed into

$$
L\left[P_{12}\right] \approx h^{2} g_{x}^{2}(F h+6 k) \frac{\partial^{2} P_{12}}{\partial \xi^{2}}+h g_{x} \frac{\partial P_{12}}{\partial \xi} .
$$

This implies that the component of $P_{12}$ arising from the right-hand side of (14) will contain terms causing the ratio $P_{12} / P_{10}$ to become unbounded as $\xi \rightarrow \infty$, unless the functions $F, G$ and $g$ are chosen to satisfy the equations

$$
\frac{\partial}{\partial x}(F h)=0, \quad \frac{\partial}{\partial x}\left[\frac{1}{h g_{x}(F h+6 k)}\right]=0,
$$

and

$$
h \frac{\partial}{\partial x}\left[\frac{h(G-F)}{h F+6 k}\right]+h \frac{\partial}{\partial x}\left[\frac{h}{h F+6 k}\right]-\frac{6 k h}{h F+6 k} h_{x}=0 .
$$

Solving Equations (15) and (16) gives

$$
\begin{aligned}
& F(x, z)=\frac{K_{1}(z)}{h(x, z)}, \\
& g(x, z)=\frac{\int_{0}^{x} h(t, z)^{-1} d t}{\int_{0}^{1} h(t, z)^{-1} d t}, \\
& G(x, z)=\frac{K_{2}(z)-6 k \ln h(x, z)}{h(x, z)},
\end{aligned}
$$


where $K_{1}(z)$ and $K_{2}(z)$ are arbitrary functions of $z$ alone. This converts Equation (11) to

$$
P_{10}+\frac{K_{1}+6 k}{h} \ln \left[\frac{K_{1}}{h}-P_{10}\right]=-\frac{\Lambda \int_{x}^{1} h(t, z)^{-1} d t}{h}+\frac{K_{2}-6 k \ln h}{h} .
$$

Applying the boundary condition at $x=1$ to $P_{10}$ gives

$$
K_{2}(z)=h(1, z)+\left(K_{1}+6 k\right) \ln \left[\frac{K_{1}}{h(1, z)}-1\right]+6 k \ln h(1, z) .
$$

The boundary condition at $x=0$ and (17) shows that to leading order in small $\Lambda^{-1}, K_{1}=h(0, z)$. Equation (18) then gives $K_{2}(z)$.

Thus, a leading order approximation to the pressure throughout the whole of Region 1 is given by $p_{10}(x, z, \Lambda)$, defined implicitly by

$$
\begin{aligned}
& p_{10}(x, z, \Lambda)+\frac{h(0, z)+6 k}{h(x, z)} \ln \left|\frac{\frac{h(0, z)}{h(x, z)}-p_{10}(x, z, \Lambda)}{\frac{h(0, z)}{h(1, z)}-1}\right| \\
= & \frac{h(1, z)}{h(x, z)}+\frac{6 k}{h(x, z)} \ln \left(\frac{h(1, z)}{h(x, z)}\right)-\frac{\Lambda \int_{x}^{1} h(t, z)^{-1} d t}{h(x, z)} .
\end{aligned}
$$

Note that $p_{10}(x, 1 / 2, \Lambda) \neq 1$; that is, the boundary condition at the edge $z=1 / 2$ is not attained. The correction required to achieve this is to be found in the $\mathcal{O}\left(B^{-1} \Lambda^{-1 / 2}\right)$ side layer mentioned earlier. A similar observation holds at the edge $z=-1 / 2$.

\section{Side layers: analysis in Regions 2 and 3}

Near the edge $z=-1 / 2$, that is, in Region 3, the stretching transformation

$$
\eta=\left(\frac{1}{2}+z\right) B \Lambda^{1 / 2}
$$


is applied to Equation (1). This yields the partial differential equation for $P_{3}(x, z, \eta, \Lambda)$, the pressure in Region 3 , as

$$
\begin{aligned}
& \Lambda^{-1} \frac{\partial}{\partial x}\left[h^{3} P_{3}\left(1+\frac{6 k}{h P_{3}}\right) \frac{\partial P_{3}}{\partial x}\right]+\frac{\partial}{\partial \eta}\left[h^{3} P_{3}\left(1+\frac{6 k}{h P_{3}}\right) \frac{\partial P_{3}}{\partial \eta}\right] \\
& =\frac{\partial}{\partial x}\left(h P_{3}\right) .
\end{aligned}
$$

From the leading order terms of (21), the differential equation determining $P_{30}(x, z, \xi, \eta)$, the leading order approximation to $P_{3}$, is

$$
\frac{\partial}{\partial \eta}\left[h^{3}\left(x,-\frac{1}{2}\right)\left(P_{30}+\frac{6 k}{h\left(x,-\frac{1}{2}\right)}\right) \frac{\partial P_{30}}{\partial \eta}\right]=\frac{\partial}{\partial x}\left[h\left(x,-\frac{1}{2}\right) P_{30}\right] .
$$

Equation (22) may be solved by the method of invariances to a leading order approximation (as the exact solution will not satisfy the boundary conditions along $z=-1 / 2)$.

Thus, a leading order approximation to the pressure in Region 3 is given by $p_{30}(x, z, \Lambda, B)$, defined implicitly by

$$
p_{30}+\left(1+\frac{6 k}{h\left(0,-\frac{1}{2}\right)}\right) \ln \left|\frac{h\left(0,-\frac{1}{2}\right)-h\left(x,-\frac{1}{2}\right) p_{30}}{h\left(0,-\frac{1}{2}\right)-h\left(x,-\frac{1}{2}\right)}\right|=-\frac{\left(\frac{1}{2}+z\right) B \Lambda^{1 / 2}}{h\left(0,-\frac{1}{2}\right)}+1 .
$$

Note that $p_{30}=1$ when $z=-1 / 2$ for all $x$. Further, for points outside the layer at $z=-1 / 2, p_{30} \rightarrow h(0,-1 / 2) / h(x,-1 / 2)$ as $B \Lambda^{1 / 2} \rightarrow \infty$; that is, the value of $p_{10}$ locally outside the trailing edge layer at $x=1$. The only discrepancy occurs within both the trailing $(x=1)$ layer and the side $(z=-1 / 2)$ layer, where it displays an exponential $\exp \left[-(z+1 / 2) \Lambda B^{1 / 2}\right]$ type structure.

Similarly, the leading order approximation to the pressure in Region 2 is given by $p_{20}(x, z, \Lambda, B)$, defined implicitly by

$$
p_{20}+\left(1+\frac{6 k}{h\left(0, \frac{1}{2}\right)}\right) \ln \left|\frac{h\left(0, \frac{1}{2}\right)-h\left(x, \frac{1}{2}\right) p_{20}}{h\left(0, \frac{1}{2}\right)-h\left(x, \frac{1}{2}\right)}\right|=-\frac{\left(\frac{1}{2}-z\right) B \Lambda^{1 / 2}}{h\left(0, \frac{1}{2}\right)}+1 .
$$




\section{Overall representation for the pressure}

A leading order representation $p_{0}(x, z, \Lambda, B)$ for the pressure field over the whole bearing may be obtained from the "sum minus common parts" rule as

$$
\begin{aligned}
p_{0}(x, z, \Lambda, B)= & p_{10}(x, z, \Lambda, B)+p_{20}(x, z, \Lambda, B)+p_{30}(x, z, \Lambda, B) \\
& -\frac{h\left(0, \frac{1}{2}\right)}{h\left(x, \frac{1}{2}\right)}-\frac{h\left(0,-\frac{1}{2}\right)}{h\left(x,-\frac{1}{2}\right)},
\end{aligned}
$$

where $p_{10}, p_{20}$ and $p_{30}$ are given by (19), (24) and (23) respectively. It is important to investigate to what degree $p_{0}$ represents the pressure over the whole bearing region $0 \leq x \leq 1,-\frac{1}{2} \leq z \leq \frac{1}{2}$.

A rearrangement of $(25)$ gives

$$
p_{0}(x, z, \Lambda, B)=p_{10}+\left(p_{20}-\frac{h\left(0, \frac{1}{2}\right)}{h\left(x, \frac{1}{2}\right)}\right)+\left(p_{30}-\frac{h\left(0,-\frac{1}{2}\right)}{h\left(x,-\frac{1}{2}\right)}\right) .
$$

Throughout Region 1, the second and third terms on the right side of (26) are subject to estimates $\mathcal{O}\left(\exp \left[-c\left(\frac{1}{2} \mp z\right) B \Lambda^{1 / 2}\right]\right)$ respectively for some $c>0$; and thus are exponentially small as $\Lambda, B \rightarrow \infty$ for all $z$ bounded away from the side layers at $z=\frac{1}{2}$ and $z=-\frac{1}{2}$. Thus, in Region $1, p_{0}(x, z, \Lambda, B)=$ $p_{10}(x, z, \Lambda, B)$ to leading order as $\Lambda \rightarrow \infty$, for all such $z$ values.

Now, (25) may also be rearranged as

$$
p_{0}=p_{20}+\left(p_{30}-\frac{h\left(0,-\frac{1}{2}\right)}{h\left(x,-\frac{1}{2}\right)}\right)+\left(p_{10}-\frac{h\left(0, \frac{1}{2}\right)}{h\left(x, \frac{1}{2}\right)}\right) .
$$

In the side layer at $z=\frac{1}{2}$, the second term on the right hand side of (27) is exponentially small as $\Lambda, B \rightarrow \infty$, while the third satisfies an estimate of the form $\mathcal{O}(\exp [-c(1-x) \Lambda])$ for some $c>0$ independent of $\Lambda$. Thus, it is small for all $x$ bounded away from $x=1$; that is, $p_{0}(x, z, \Lambda)=p_{20}(x, z, \Lambda)$ to leading order as $\Lambda, B \rightarrow \infty$, in the side layer at $z=\frac{1}{2}$, and for all $x$ outside the trailing layer at $x=1$. 
Similar arguments show that $p_{0}(x, z, \Lambda)=p_{30}(x, z, \Lambda)$ to leading order as $\Lambda, B \rightarrow \infty$ in the side layer at $z=-\frac{1}{2}$ and for $x$ bounded away from $x=1$.

If it is accepted that the approximations $p_{10}, p_{20}$ and $p_{30}$ represent the pressure field on their various sub domains (based as they are on equations approximating the full equation (1) on these domains), then the above results lead to the conclusion that $p_{0}(x, z, \Lambda, B)$, as defined by (25), represents the pressure field in the bearing to leading order, except in neighbourhoods of the corners at $(x, z)=(1, \pm 1 / 2)$, where $p_{0}$ may differ from the pressure by amounts that display a characteristic exponential decay away from the boundaries $x=1, z= \pm 1 / 2$.

\section{Discussion}

The expression (25) provides a convenient leading order approximation to the pressure field over virtually all of the bearing region. The only exceptions are the corner regions at $(x, z)=(1, \pm 1 / 2)$; and even then, the discrepancy is not uniformly significant. These corner regions, at the intersections of the side and trailing layers, are of dimensions $\mathcal{O}\left(\Lambda^{-1}\right) \times \mathcal{O}\left(B^{-1} \Lambda^{-1 / 2}\right)$ as $\Lambda \rightarrow \infty$, $B \rightarrow \infty$. Thus, the integrated effect of the pressure over these regions is $\mathcal{O}\left(B^{-1} \Lambda^{-3 / 2}\right)$. This has implications for the load - the load bearing capacity of the bearing, given by the integral of the pressure over the whole bearing area. This is not calculated here [1].

While the component functions $p_{10}, p_{20}$ and $p_{30}$ are given implicitly, their evaluation and graphical representation poses no real difficulty. For each $(x, z)$ pair, the appropriate implicit equation may be solved numerically for the corresponding $p$ value, using a Newton-Raphson or similar elementary procedure. The resulting $(x, z, p)$ triplets may then be plotted using standard software. For longitudinal or cross sections as presented here, the appropriate $p-x$ or $p-z$ relationship may be plotted using a standard package such as Maple's implicitplot. 


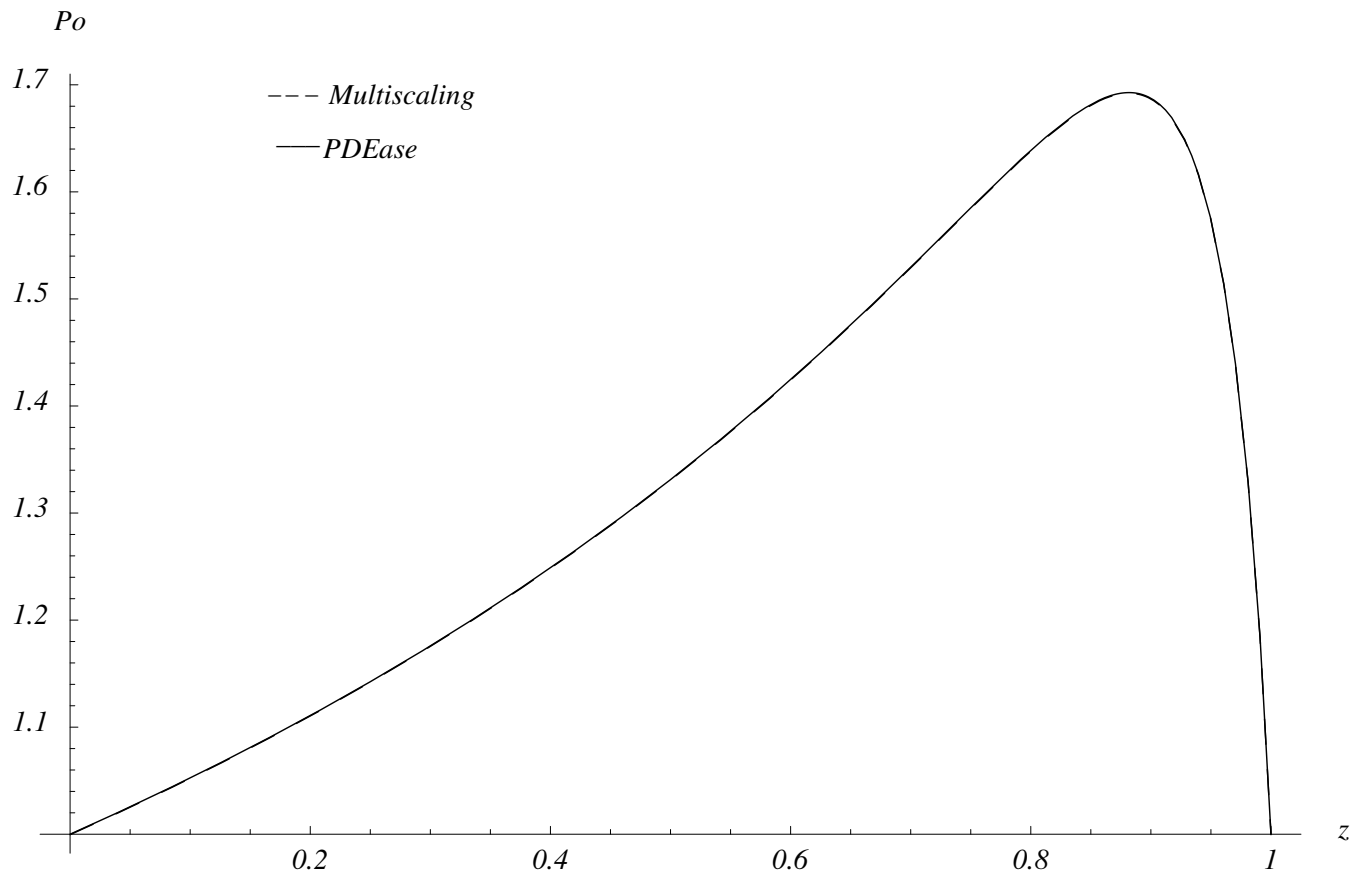

Figure $3:$ Midline pressure distribution for $h=1-0.5 x, 6 k=1, \Lambda \approx 200$, $B \approx 2.9$, as approximated by (25) and PDEase. 


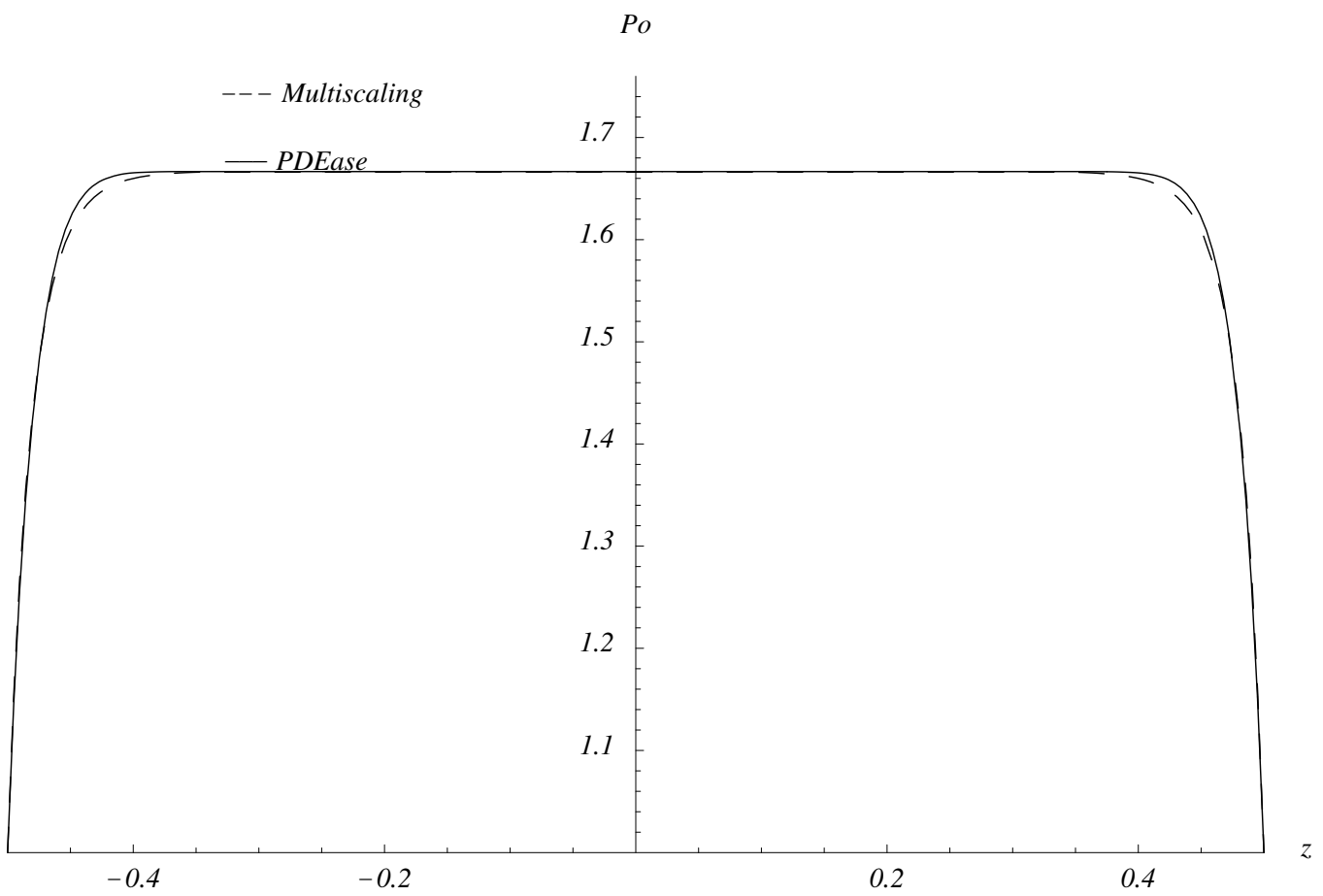

Figure 4: Transverse pressure distribution at $x=0.8$ for $h=1-0.5 x$, $6 k=1, \Lambda \approx 200, B \approx 2.9$, as approximated by (25) and PDEase.

Figure 3 compares the pressure profile along the bearing midline $(z=0)$ for a linear profile, as computed using (25) with the results of a computation using the finite element package PDEase. It is clear that the difference between the two results is insignificant.

Figure 4 compares the results of using (25) with that of PDEase for the same linear profile for a section across the bearing at $x=0.8$. Again, the agreement is very good, with, as expected, a small discrepancy in the side layer at $z= \pm 1 / 2$. Figure 5 shows a three-dimensional plot of the pressure field as given by the approximate relation (25) over the whole bearing area. 


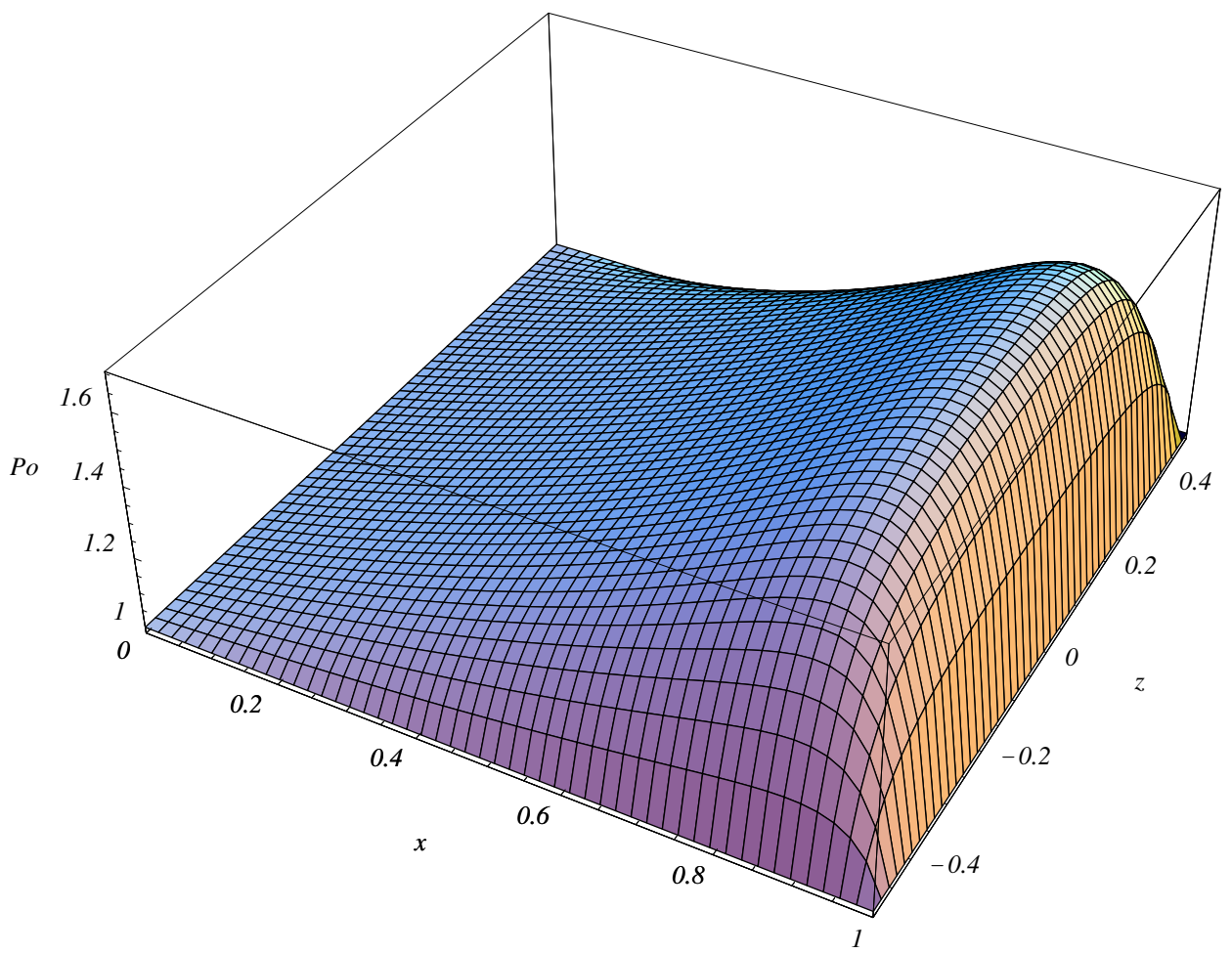

Figure 5: Pressure distribution for $h=1-0.5 x, 6 k=1, \Lambda \approx 200, B \approx 2.9$, as approximated by $(25)$. 
Both the trailing edge layer and side layers are clearly displayed.

In closing, note that the perturbation analysis above computed a leading order approximation to the pressure throughout the bearing. Extension to higher order terms would require computation of the $\mathcal{O}\left(B^{-2}\right)$ and $\mathcal{O}\left(\Lambda^{-1}\right)$ terms in (7), and their corresponding corrections in the side layers. This is well beyond the scope of the present paper, and is being investigated.

\section{References}

[1] Aliu E., Shepherd J. J. and Connell H. J., Multiscaling analysis of a nonlinear boundary value problem in lubrication theory, Int. J. Appl. Mech. and Engrg, 9, No. 3, (2004), pp. 455-470 C480, C487

[2] Gross W. A. and Zachmanoglou E. C., Perturbation solutions for gas-lubricating films, J. Bas. Eng., TRANS. ASME, Series D, 83, No. 1, Mar. 1961, pp. 139-144. C478

[3] Burgdorfer A., The influence of the molecular mean free path on the performance of hydrodynamic gas lubricated bearings, J. Bas. Eng. TRANS. ASME, Series D, March 1959, pp. 94-100. C477

[4] Reynolds O., On the theory of lubrication and its application to Mr. Beauchamp Tower's experiments, Phil. Trans. Roy Soc. (London), Ser. A, 177, 1886, pp. 154-234. C477

[5] Schmitt J. A., Asymptotic methods in gas lubrication theory for slider bearings, Ph.D. Thesis, Rensselaer Polytechnic Institute, Troy, N. Y. June 1975. C479

[6] Schmitt J. A. and DiPrima R. C., Asymptotic methods for a general finite width gas slider bearing, ASME J. Lub. Tech., 100, 1978, pp. 254-260. C479 